\title{
Development and validation of a Surveillance, Epidemiology, and End Results (SEER)-based prognostic nomogram for predicting survival in elderly patients with gastric cancer after surgery
}

\author{
Yujie Zhang ${ }^{1 \#}$, Chaoran Yu ${ }^{2,3 \#}$ \\ ${ }^{1}$ Department of Gastrointestinal Surgery, Tongji Hospital, Tongji Medical College in Huazhong University of Science and Technology, Wuhan, \\ China; ${ }^{2}$ Fudan University Shanghai Cancer Center, Fudan University, Shanghai, China; ${ }^{3}$ Department of Oncology, Shanghai Medical College, Fudan \\ University, Shanghai, China \\ Contributions: (I) Conception and design: All authors; (II) Administrative support: All authors; (III) Provision of study materials or patients: All \\ authors; (IV) Collection and assembly of data: All authors; (V) Data analysis and interpretation: All authors; (VI) Manuscript writing: All authors; (VII) \\ Final approval of manuscript: All authors. \\ "These authors contributed equally to this work. \\ Correspondence to: Yujie Zhang. Department of Gastrointestinal Surgery, Tongji Hospital, Tongji Medical College in Huazhong University of Science \\ and Technology, Wuhan, China. Email: yujiezhang@outlook.com.
}

Background: Elderly gastric cancer (ELGC) remains one of the intensively investigated topics during the last decades. To establish a comprehensive nomogram for effective clinical practice and assessment is of significance. This study is designed to develop a prognostic nomogram for ELGC both in overall survival (OS) and cancer-specific survival (CSS).

Methods: The recruited cases were from the Surveillance, Epidemiology, and End Results (SEER) database and input for the construction of nomogram.

Results: A total of 4,414 individuals were recruited for this study, of which 2,208 were randomly in training group and 2,206 were in validation group. In univariate analysis of $\mathrm{OS}$, significant variables $(\mathrm{P}<0.05)$ included age, marital status, grade, American Joint Committee on Cancer (AJCC) tumor-node-metastasis (TNM) stage, bone/brain/liver/lung metastasis and tumor size. In univariate analysis of CSS, significant variables $(\mathrm{P}<0.05)$ included age, grade, AJCC TNM stage, bone/brain/liver/lung metastasis and tumor size. In multivariate analysis of OS, sex, age, race, grade, TNM stage, lung metastasis and tumor size were considered as the significant variables and subjected to the establishment of nomogram. In multivariable analysis of CSS, age, grade, TNM, tumor size were considered as the significant variables and input to the establishment of nomogram. Sex, age, race, grade, TNM stage, lung metastasis and tumor size were included for the establishment of nomogram in OS while age, grade, TNM, tumor size were included to the establishment of nomogram in CSS. C-index, decision curve analysis (DCA) and the area under the curve (AUC) showed distinct value of newly established nomogram models. Both OS and CSS nomograms showed higher statistic power over the AJCC stage.

Conclusions: This study established and validated novel nomogram models of OS and CSS for ELGC based on population dataset.

Keywords: Elderly gastric cancer (ELGC); nomogram; overall survival (OS); cancer-specific survival (CSS)

Submitted Nov 21, 2020. Accepted for publication Mar 05, 2021.

doi: 10.21037/jgo-20-536

View this article at: http://dx.doi.org/10.21037/jgo-20-536 


\section{Introduction}

Gastric cancer (GC) is the fifth most common malignancies worldwide with approximately 783,000 deaths in 2018, ranking third in deadly cancer (1-3). Both incidence and mortality of GC are featured by geological, diet and infection factors. Improved knowledge of prevention on Helicobacter pylori infection and related risk factors of GC have considerably reduced the overall incidence $(4,5)$. However, increasing incidence of GC in cardia has been noticed during the past decades up to over 7-fold as many (1). Moreover, several classification systems, such as Lauren classification and an alternative one (papillary/tubular/mucinous/poorly cohesive), do not fully demonstrate clinical utility (6).

Increasing notice of heterogeneity intrigues the genomic exploration of GC, which is of essence to the full picture. The Cancer Genome Atlas (TCGA) study revealed that, comparing to classic tumor-node-metastasis (TNM) classification from WHO system, GC could be divided into four groups, including Epstein-Barr virus (EBV) positive (recurrent PIK3CA mutation and extreme DNA hypermethylation), microsatellite unstable (MSI, high mutation rates), genomically stable and chromosomal instability groups $(6,7)$. Interestingly, TCGA indicates that genomically stable GC are more enriched at an earlier age (median age 59 years) whereas MSI tumors are found at a relatively older age (median age 72 years) (7). Another nation-wide investigation reports that age-specific trends vary significantly between older and younger groups across the United States (8). Incidence (per 100,000) drops significantly from 19.8 to 12.8 in GC patients with age 60 to 84 while increases from 0.27 to 0.45 in GC with age 25 to 39 years (8). Our previous study reported an effective prognostic nomogram for young GC (9). Only tumor size and tumor site were included for the establishment of nomogram in this study given the comparably limited sample size (276 cases as training set) (9).

Elderly GC (ELGC) remains one of the intensively investigated topics during the last decades. It is defined as GC patients over 70 years (10-12). ELGC is more correlated to intestinal type, expansive tumor growth and synchronous multiplicity with comparable poor survival outcome (11). Another study indicates that ELGC is more likely to present advanced TNM stage and larger tumor size with less odds in distant metastasis (12). Therefore, to establish a comprehensive nomogram for effective clinical practice and assessment is of significance. Noteworthy, Roberto et al. reported a prognostic nomogram for ELGC using 143 cases aged over 80 years (13). Given the shortage of large sample size in GC, it is more reliable to conduct a prognostic nomogram using public-available Surveillance, Epidemiology, and End Results (SEER) database from the National Cancer Institute (14-16). Therefore, in this study, ELGC cases from SEER database were retrieved for the establishment of nomogram. We present the following article in accordance with the TRIPOD reporting checklist (available at http://dx.doi.org/10.21037/jgo-20-536).

\section{Methods}

The study was conducted in accordance with the Declaration of Helsinki (as revised in 2013).

\section{Input data from SEER database}

All the ELGC cases were retrieved from the SEER database (2004-2016) using ID 16595-Nov2019 (17). The criteria of inclusion were as follow: (I) stomach confirmed in "Site and morphology, site recode ICD-3/WHO 2008”; (II) age above 70 (including); (III) complete American Joint Committee on Cancer (AJCC) TNM information; (IV) only one primary tumor; (V) with surgery performed. Moreover, histologic type ICD-O-3 was retrieved to not only adenocarcinoma but other types of malignancies were covered, such as mixed tumor (malignant NOS). Clinical information, including age, sex and numerous variables were collected for characterization and further univariate/multivariate analysis. Total ELGC cases were randomized into training and validation groups equally. Overall survival (OS) and cancer-specific survival (CSS) were listed as primary and secondary endpoints, respectively. Moreover, $\mathrm{x}$-tile program (Yale University, United States) was utilized for the optimal cutoff value of consecutive variables, including age and tumor size (18).

\section{Statistical analysis}

Univariate and multivariate analysis of all recorded clinical variables was performed. Those variables with statistically significance were extracted for the establishment of nomogram model both in OS and CSS. Next, the validation group was analyzed for assessment using the concordance index (C-index) and calibration plot. Moreover, the predict power of this nomogram was also assessed by ROC and decision curve analysis (DCA) curves. All analysis was 


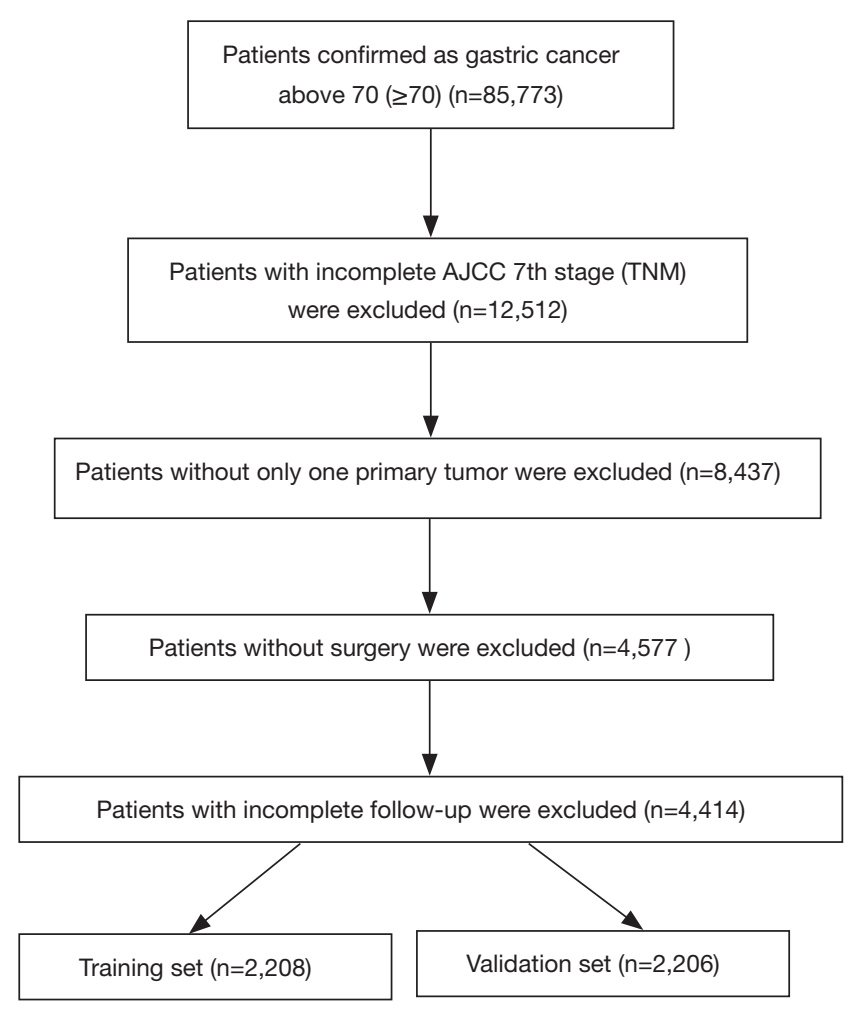

Figure 1 The inclusion flowchart of recruited ELGC patients. ELGC, elderly gastric cancer. performed by R software 3.6.0 (Vienna, Austria).

\section{Results}

\section{Patient recruitment and characterization of cases}

A total of 4,414 individuals with complete TNM stage and follow-up were recruited for this study, of which 2,208 were randomly in training group and 2,206 were in validation group (Figure 1). The cutoff value of age was 75 and 85 years (Figure $2 A, B, C$ ). The cutoff value of tumor size was 25 and $47 \mathrm{~mm}$ (Figure 2D,E,F). Among the recruited cases, 2,495 were male and 1,919 were females. Four hundred ninety-one individuals were elder than 86 years old $(\geq 86)$, 1,894 were younger than 75 years old $(\leq 75), 2,029$ were between 76-85 years old. There were 1,256 individuals in $\mathrm{T} 1,700$ in $\mathrm{T} 2,1,456$ in $\mathrm{T} 3$ and 1,002 in T4. There were 2,413 individuals in N0, 783 in N1, 534 in N2 and 684 in N3. There were 4,075 individuals in distant metastasis negative and 339 in distant metastasis positive. There were only 8 positive individuals in bone metastasis, 36 in brain metastasis, 126 in liver metastasis and 26 in lung metastasis. Baseline variables were characterized between each group without significant difference (Table 1).

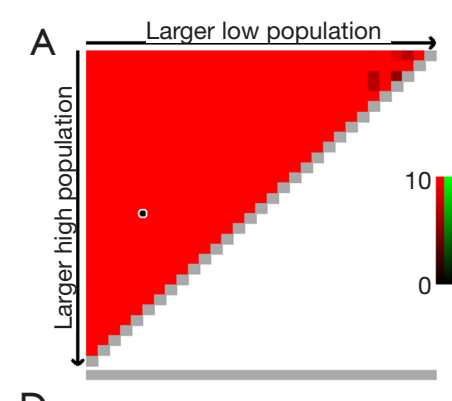

$\mathrm{D}$
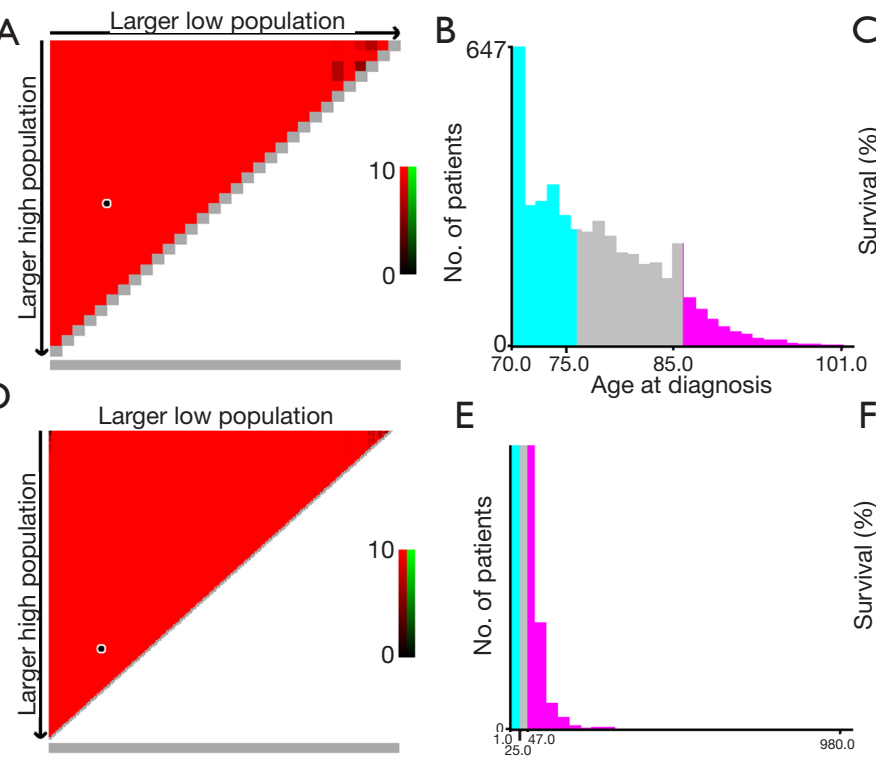

$\mathrm{E}$

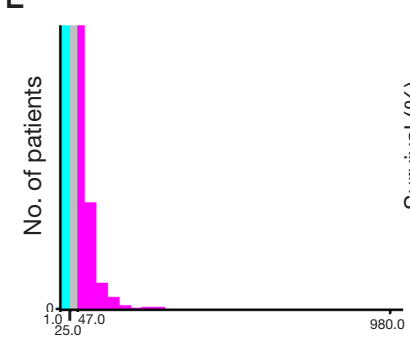

CS tumor size (2004-2015)
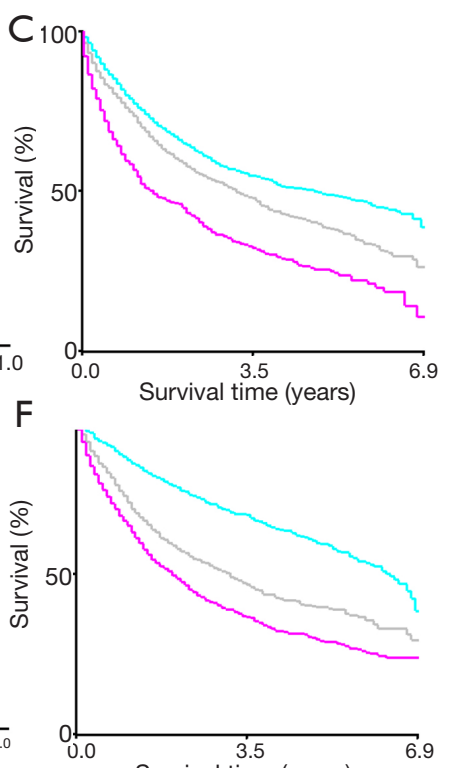

Survival time (years)

Figure 2 Determination of best-cutoff points of age and tumor size variables by the X-tile software. (A) Identification of optimal cutoff point using X-tile plot of training sets in age; (B) the cutoff points were displayed in histogram; (C) distinct prognosis among high/middle/ low subsets using a Kaplan-Meier plot (low subset = blue, middle subset = gray, high subset = magenta); (D) identification of optimal cutoff point using X-tile plot of training sets in tumor size; (E) the cutoff points were displayed in histogram; (F) distinct prognosis among high/ middle/low subsets using a Kaplan-Meier plot (low subset = blue, middle subset = gray, high subset = magenta). CS, collaborative stage . 
Table 1 Baseline demographic and clinical characteristics of patients with elderly gastric cancer (ELGC)

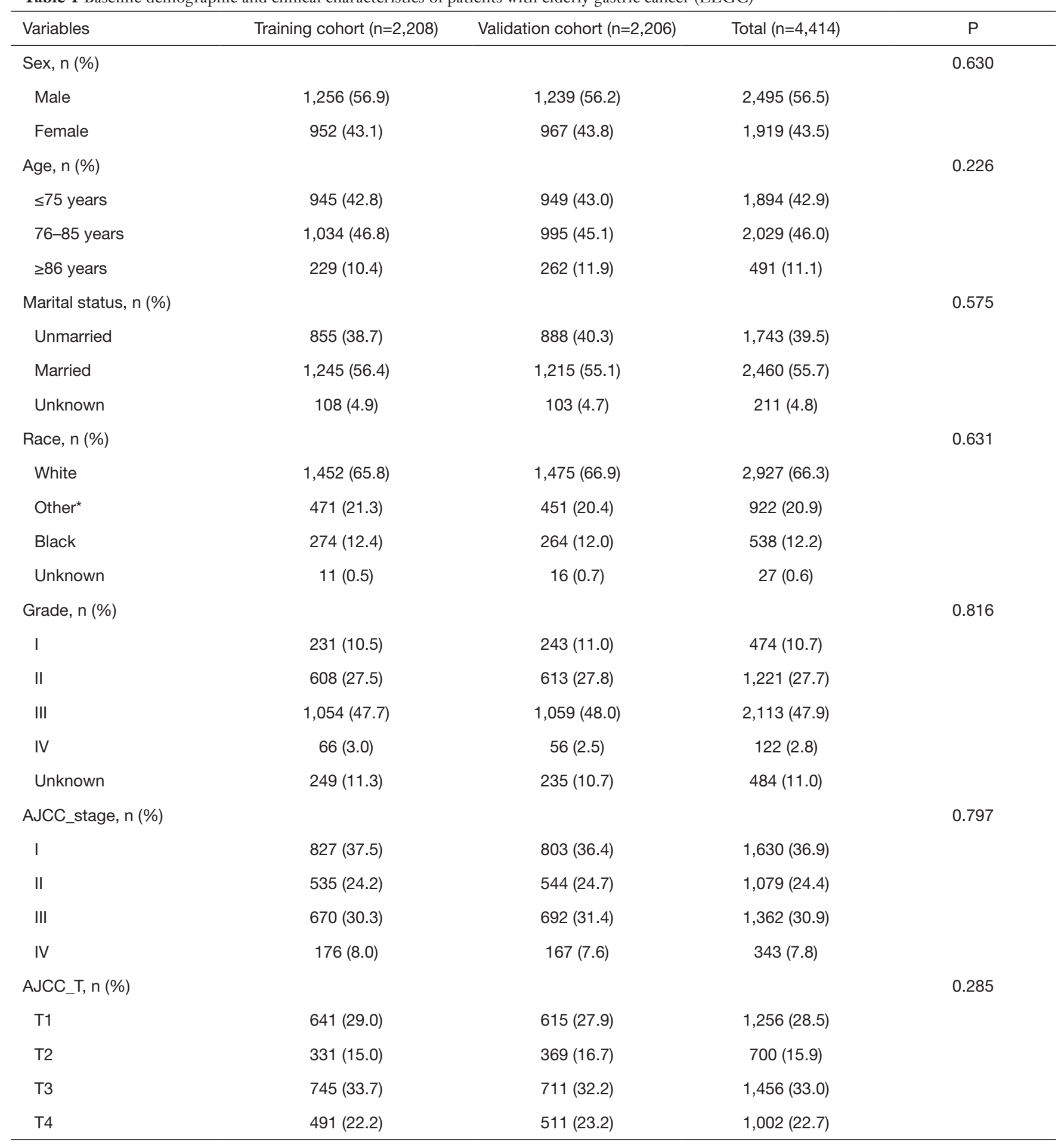

Table 1 (continued) 
Table 1 (continued)

\begin{tabular}{|c|c|c|c|c|}
\hline Variables & Training cohort $(n=2,208)$ & Validation cohort $(n=2,206)$ & Total $(n=4,414)$ & $P$ \\
\hline No & $1,219(55.2)$ & $1,194(54.1)$ & $2,413(54.7)$ & \\
\hline $\mathrm{N} 1$ & 373 (16.9) & $410(18.6)$ & $783(17.7)$ & \\
\hline N2 & $260(11.8)$ & $274(12.4)$ & $534(12.1)$ & \\
\hline AJCC_M, n (\%) & & & & 0.699 \\
\hline MO & 2,035 (92.2) & $2,040(92.5)$ & $4,075(92.3)$ & \\
\hline M1 & $173(7.8)$ & $166(7.5)$ & $339(7.7)$ & \\
\hline Bone metastasis, $\mathrm{n}(\%)$ & & & & 0.769 \\
\hline Unknown & $19(0.9)$ & $20(0.9)$ & $39(0.9)$ & \\
\hline Brain metastasis, $\mathrm{n}(\%)$ & & & & 0.485 \\
\hline No & 2,191 (99.2) & $2,186(99.1)$ & 4,377 (99.2) & \\
\hline Yes & $16(0.7)$ & $20(0.9)$ & $36(0.8)$ & \\
\hline Unknown & $1(0.0)$ & $0(0.0)$ & $1(0.0)$ & \\
\hline Liver metastasis, n (\%) & & & & 0.297 \\
\hline No & 2,126 (96.3) & $2,133(96.7)$ & $4,259(96.5)$ & \\
\hline Yes & $70(3.2)$ & $56(2.5)$ & $126(2.9)$ & \\
\hline Tumor size, $\mathrm{n}(\%)$ & & & & 0.999 \\
\hline$\leq 2.5 \mathrm{~cm}$ & $602(27.3)$ & $600(27.2)$ & $1,202(27.2)$ & \\
\hline $2.6-4.7 \mathrm{~cm}$ & $558(25.3)$ & $555(25.2)$ & $1,113(25.2)$ & \\
\hline$\geq 4.8 \mathrm{~cm}$ & $805(36.5)$ & 805 (36.5) & $1,610(36.5)$ & \\
\hline Unknown & $243(11.0)$ & $246(11.2)$ & $489(11.1)$ & \\
\hline
\end{tabular}

Other*: American Indian/AK Native, Asian/Pacific Islander.

\section{Establishment of nomogram models}

In univariate analysis of $\mathrm{OS}$, significant variables $(\mathrm{P}<0.05)$ included age, marital status, grade, AJCC TNM stage, bone/brain/liver/lung metastasis and tumor size. In univariate analysis of CSS, significant variables $(\mathrm{P}<0.05)$ included age, grade, AJCC TNM stage, bone/brain/liver/ lung metastasis and tumor size (Table 2). However, to fully identify potential variables that may contribute to the final outcome, variables with $\mathrm{P}<0.2$ were included for multivariable analysis, separately (Table 3). In multivariate 
Table 2 Univariate cox regression analysis of overall survival and cancer-specific survival in the training cohort

\begin{tabular}{|c|c|c|c|c|}
\hline Variables & \multicolumn{2}{|c|}{ Overall survival } & \multicolumn{2}{|c|}{ Cancer-specific survival } \\
\hline \multicolumn{5}{|l|}{ Sex } \\
\hline Male & Reference & & Reference & \\
\hline Female & $0.919(0.816-1.035)$ & 0.163 & $0.944(0.822-1.084)$ & 0.413 \\
\hline$\leq 75$ years & Reference & & Reference & \\
\hline $76-85$ years & $1.251(1.101-1.421)$ & $<0.001^{*}$ & $1.165(1.006-1.349)$ & $0.042^{*}$ \\
\hline$\geq 86$ years & 2.095 (1.748-2.512) & $<0.001^{*}$ & $1.834(1.479-2.273)$ & $<0.001^{*}$ \\
\hline \multicolumn{5}{|l|}{ Marital status } \\
\hline Unknown & $0.589(0.425-0.817)$ & $0.002^{*}$ & $0.629(0.433-0.914)$ & $0.015^{\star}$ \\
\hline \multicolumn{5}{|l|}{ Race } \\
\hline White & Reference & & Reference & \\
\hline Other & $0.892(0.768-1.036)$ & 0.133 & $0.939(0.792-1.114)$ & 0.47 \\
\hline Black & $1.119(0.938-1.334)$ & 0.213 & $1.053(0.854-1.299)$ & 0.627 \\
\hline Unknown & $2.979 \mathrm{e}-07$ (0.000-Inf) & 0.981 & $2.998 \mathrm{e}-07$ (0.000-Inf) & 0.984 \\
\hline \multicolumn{5}{|l|}{ Grade } \\
\hline I & Reference & & Reference & \\
\hline \multicolumn{5}{|l|}{ AJCC_stage } \\
\hline I & Reference & & Reference & \\
\hline II & $1.770(1.478-2.119)$ & $<0.001^{*}$ & $2.92(2.294-3.717)$ & $<0.001^{*}$ \\
\hline III & $4.332(3.702-5.069)$ & $<0.001^{*}$ & 7.87 (6.344-9.762) & $<0.001^{*}$ \\
\hline IV & $6.133(4.979-7.555)$ & $<0.001^{*}$ & 11.67 (8.998-15.137) & $<0.001^{*}$ \\
\hline \multicolumn{5}{|l|}{ AJCC_T } \\
\hline $\mathrm{T} 1$ & Reference & & Reference & \\
\hline T2 & $1.128(0.891-1.429)$ & 0.317 & $1.407(1.028-1.925)$ & $0.033^{*}$ \\
\hline T3 & $2.462(2.080-2.914)$ & $<0.001^{*}$ & 3.903 (3.114-4.894) & $<0.001^{*}$ \\
\hline $\mathrm{T} 4$ & $4.458(3.744-5.308)$ & $<0.001^{*}$ & 7.437 (5.910-9.358) & $<0.001^{*}$ \\
\hline
\end{tabular}

Table 2 (continued) 
Table 2 (continued)

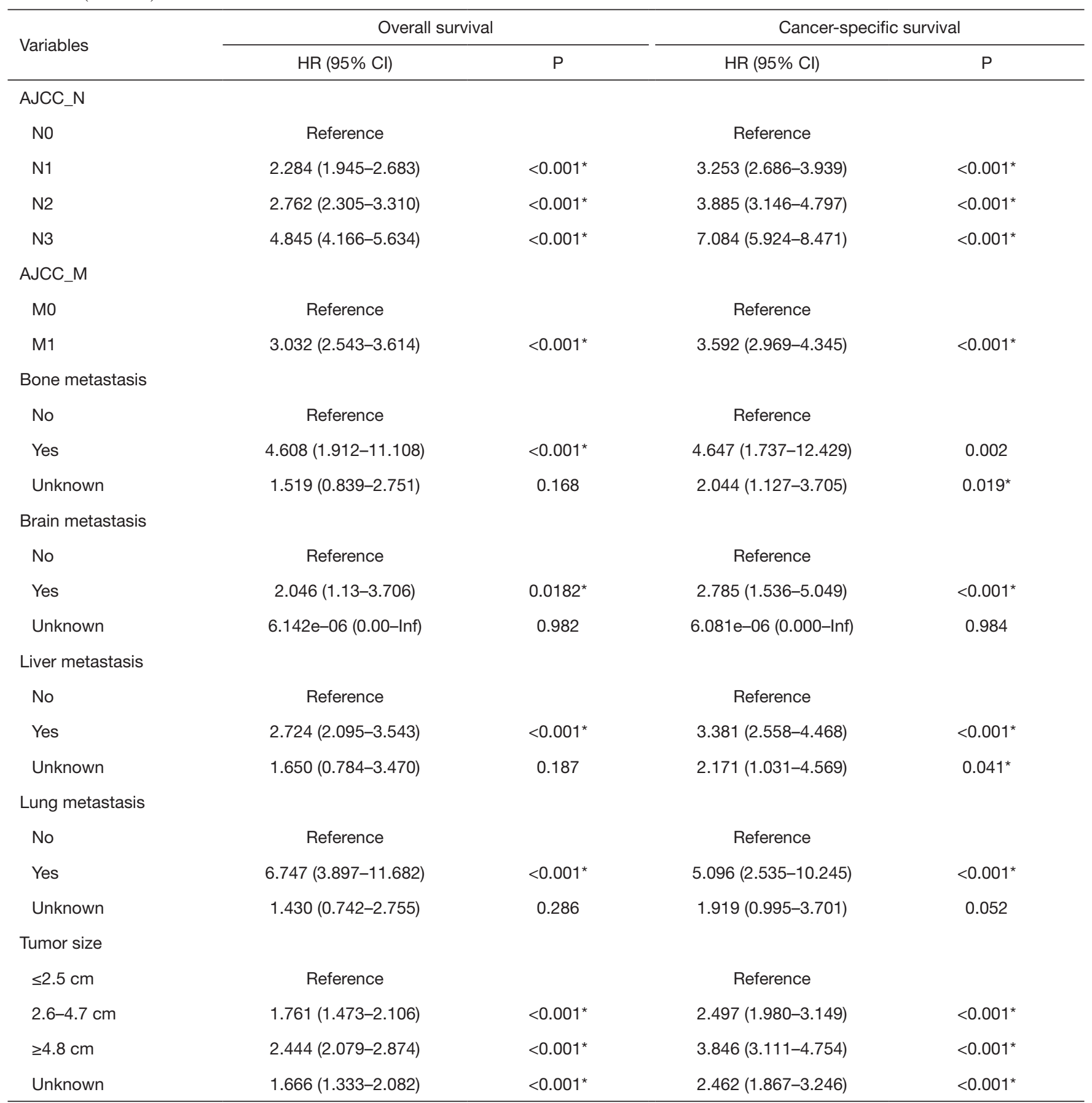

*, $\mathrm{P}<0.05$. HR, hazard ratio; $\mathrm{Cl}$, confidence interval.

analysis of OS (Figure 3), sex, age, race, grade, TNM stage, lung metastasis and tumor size were considered as the significant variables and subjected to the establishment of nomogram (Figure 4A). In multivariable analysis of CSS
(Figure 3B), age, grade, TNM, tumor size were considered as the significant variables and input to the establishment of nomogram (Figure $4 B$ ). In addition, specific scores of each included variable were displayed (Table 4). 
Table 3 Multivariate cox regression analysis of overall survival and cancer-specific survival in the training cohort

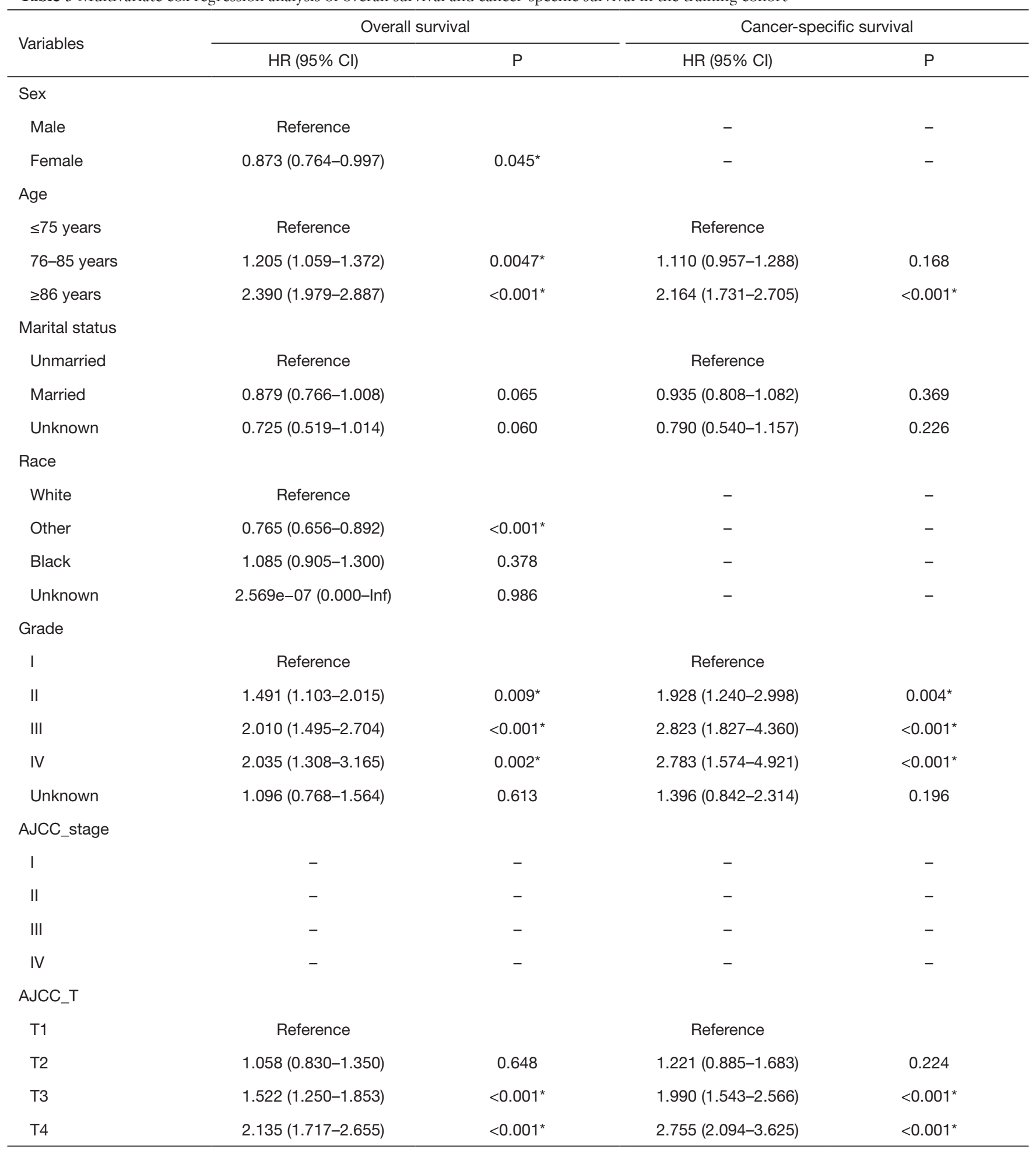

Table 3 (continued) 
Table 3 (continued)

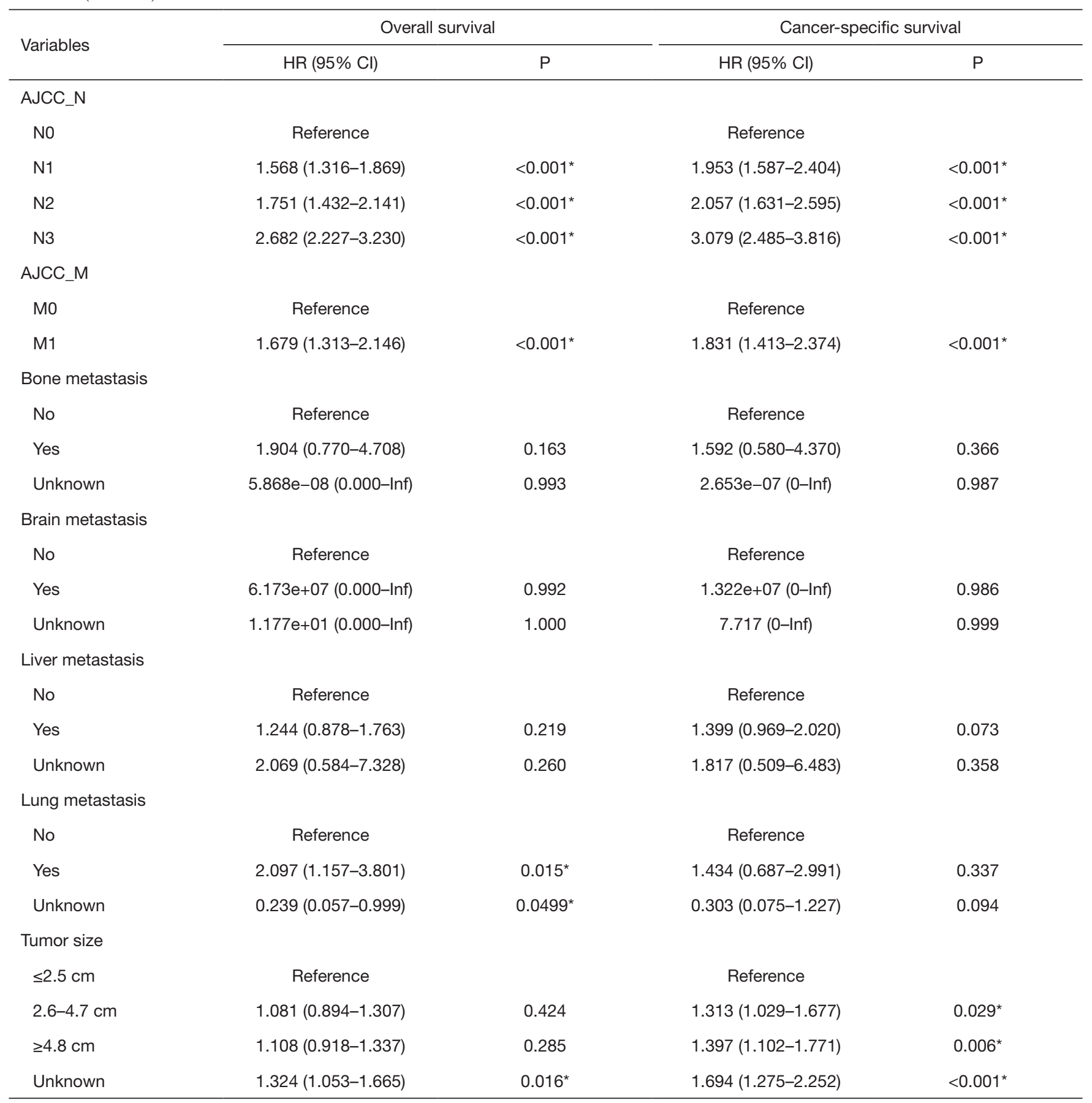

*, $\mathrm{P}<0.05$. HR, hazard ratio; $\mathrm{Cl}$, confidence interval. 


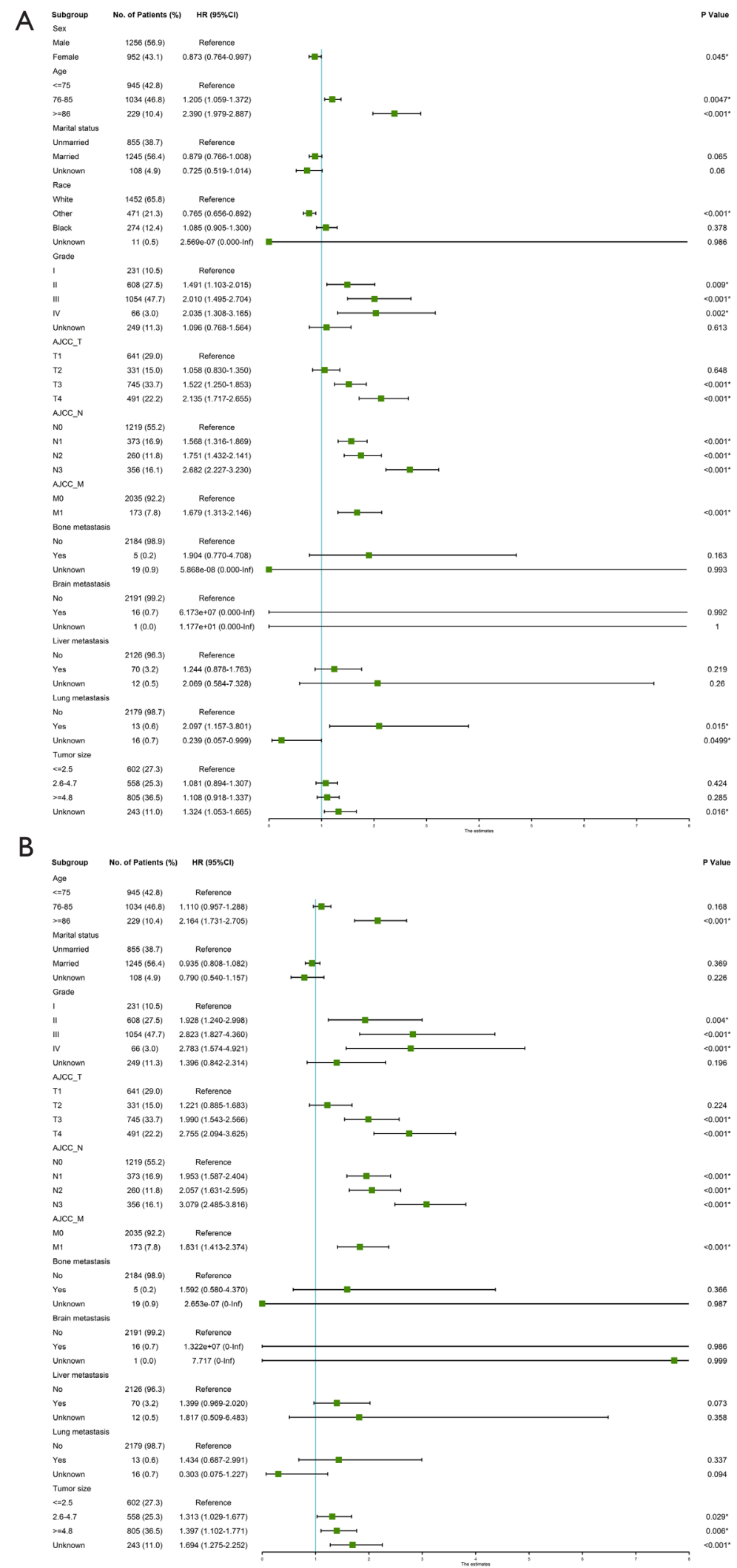

Figure 3 Forest plot of all variables with hazard ratios in ELGC. * $\mathrm{P}<0.05$. HR, hazard ratio; CI, confidence interval; ELGC, elderly gastric cancer. 


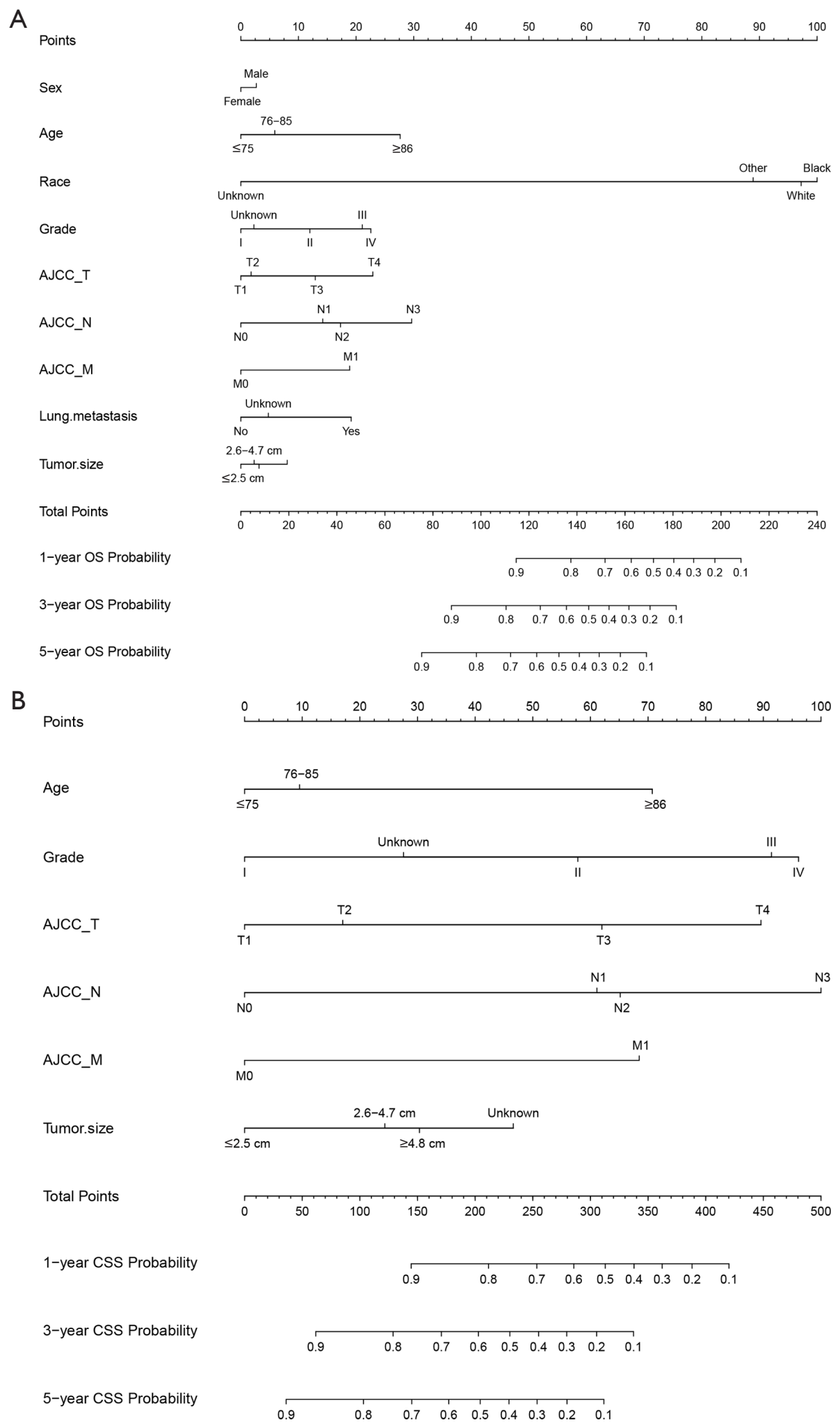

Figure 4 Establishment of nomograms regarding both overall survival (OS) and cancer-specific survival (CSS). (A) Establishment of OS nomogram; (B) establishment of CSS nomogram.

(C) Journal of Gastrointestinal Oncology. All rights reserved. 
Table 4 Scores of prognostic factors in the OS and CSS nomograms

\begin{tabular}{|c|c|c|}
\hline Characteristic & OS nomogram & CSS nomogram \\
\hline \multicolumn{3}{|l|}{ Sex } \\
\hline Male & 3 & - \\
\hline Female & 0 & - \\
\hline \multicolumn{3}{|l|}{ Age } \\
\hline$\leq 75$ years & 0 & 0 \\
\hline $76-85$ years & 6 & 10 \\
\hline$\geq 86$ years & 28 & 71 \\
\hline \multicolumn{3}{|l|}{ Race } \\
\hline White & 97 & - \\
\hline Other & 89 & - \\
\hline Black & 100 & - \\
\hline Unknown & 0 & - \\
\hline \multicolumn{3}{|l|}{ Grade } \\
\hline I & 0 & 0 \\
\hline ॥ & 12 & 58 \\
\hline III & 21 & 91 \\
\hline IV & 23 & 96 \\
\hline Unknown & 2 & 28 \\
\hline \multicolumn{3}{|l|}{ AJCC_T } \\
\hline $\mathrm{T} 1$ & 0 & 0 \\
\hline T2 & 2 & 17 \\
\hline T3 & 13 & 62 \\
\hline $\mathrm{T} 4$ & 23 & 90 \\
\hline \multicolumn{3}{|l|}{ AJCC_N } \\
\hline No & 0 & 0 \\
\hline N1 & 14 & 61 \\
\hline N2 & 17 & 65 \\
\hline N3 & 30 & 100 \\
\hline \multicolumn{3}{|l|}{ AJCC_M } \\
\hline MO & 0 & 0 \\
\hline M1 & 19 & 68 \\
\hline \multicolumn{3}{|c|}{ Lung metastasis } \\
\hline No & 0 & - \\
\hline Yes & 19 & - \\
\hline Unknown & 5 & - \\
\hline \multicolumn{3}{|l|}{ Tumor size } \\
\hline$\leq 2.5 \mathrm{~cm}$ & 0 & 0 \\
\hline $2.6-4.7 \mathrm{~cm}$ & 2 & 24 \\
\hline$\geq 4.8 \mathrm{~cm}$ & 3 & 30 \\
\hline Unknown & 8 & 47 \\
\hline
\end{tabular}

OS, overall survival; CSS, cancer-specific survival.

\section{Validation of the established nomograms}

Next, to validate the power of nomograms, C-index and calibration plots were employed (Figures 5,6). Specifically, the $\mathrm{C}$-index of OS in the training set was $0.730[95 \%$ confidence interval (CI): $0.714-0.746]$ while in the validation set was 0.735 (95\% CI: $0.719-0.751)$. The C-index of CSS in the training set was 0.765 (95\% CI: $0.748-0.782$ ) while in the validation set was 0.759 (95\% CI: 0.742-0.776) (Table 5).

In DCA, nomograms of OS and CSS performed better than AJCC TNM stage (Figure 7). In fact, the both OS and CSS nomograms showed higher statistic power over the AJCC stage (Table 5, Figures 8,9).

\section{Discussion}

By now, this is the first study highlighting the potential value of nomograms targeting ELGC with population dataset. For consecutive variables like age and tumor size, $\mathrm{x}$-tile software was utilized for the optimal cutoff. There are a few points that should be discussed prior to conclusion.

First, although univariate analysis of OS and CSS have identified variables with significance $(\mathrm{P}<0.05)$, we believe a proper extension of cutoff $\mathrm{P}$ value $(\mathrm{P}<0.2)$ is reasonable and applicable. Second, sex and marital status are not included at the nomograms of OS and CSS in this study. In fact, the role of marital status in GC had been investigated (19). Qiu et al. reported that unmarried GC tended to have higher risk of cancer specific mortality (19). Our study showed no significant role of marital status associated with ELGC, indicating a possible close association of marital status to young GC rather ELGC. Third, among all four classic distant metastasis lesions, lung, brain, bone and liver, only lung metastasis was identified as a contributor to OS nomogram while none of distant metastatic lesions was associated with CSS nomogram. A similar study focusing on the nomograms of metastatic gastric adenocarcinoma with palliative gastrectomy reported that metastasis was one of the major contributors (20). However, distant lymph node metastasis and visceral metastasis were mainly evaluated. Our study complements the finding of previous study by highlighting the role of lung metastasis in the prognosis prediction of OS.

Similarly, there were numerous studies that focused on the age of GC using nomograms (21). Zhou et al. reported a prognostic nomogram to predict the OS of elderly GC using SEER data resource. However, there are several 
A

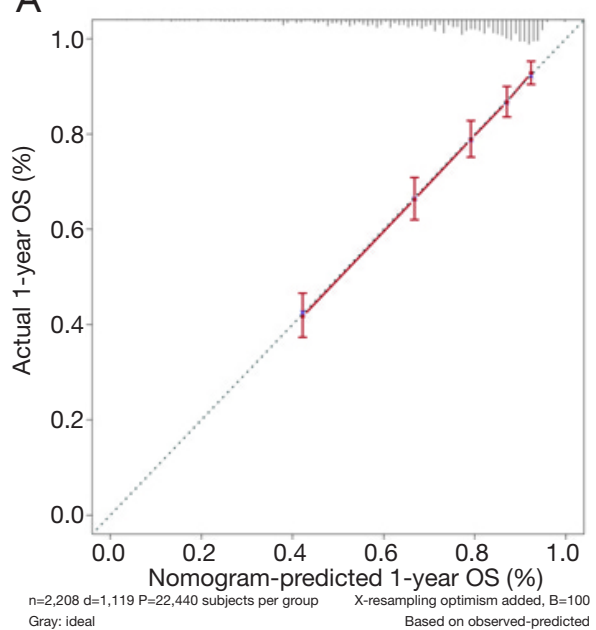

D

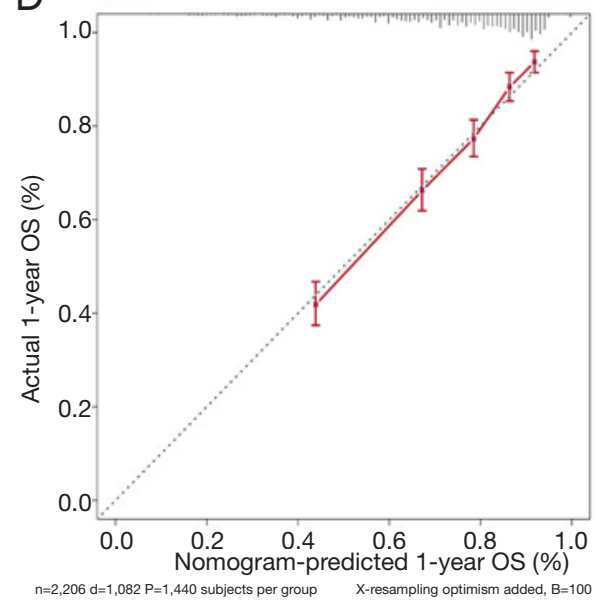

B

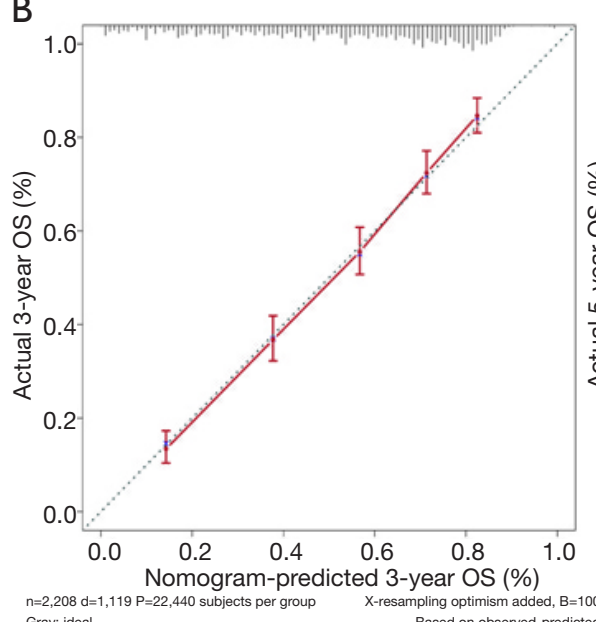

$\mathrm{E}$

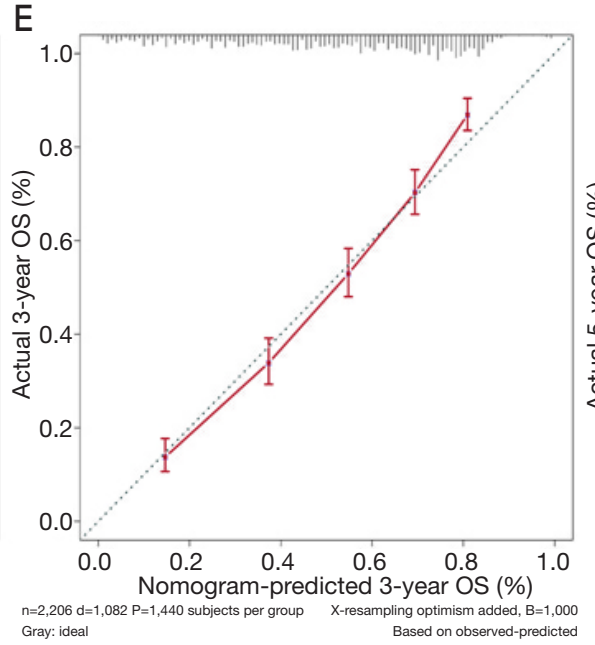

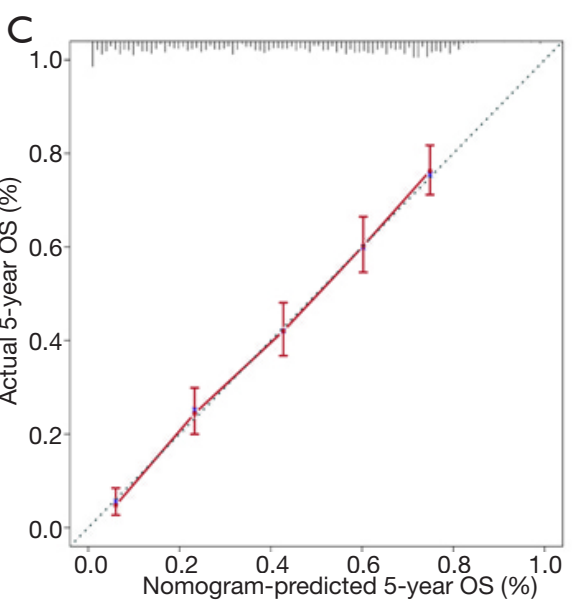

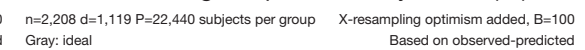

$\mathrm{F}$

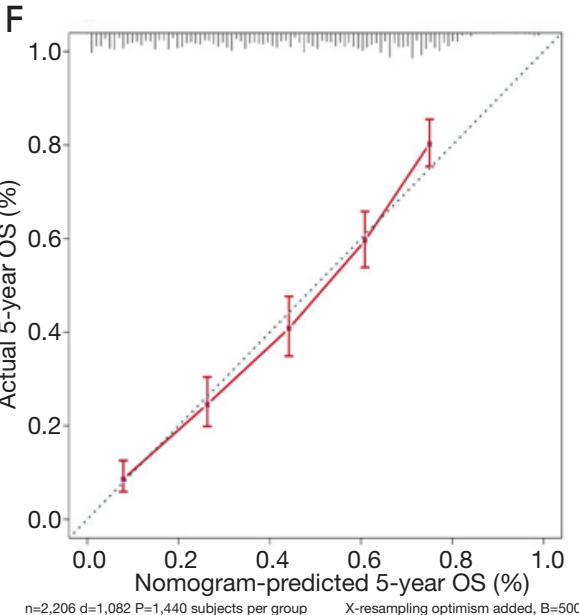

Figure 5 Evaluation of calibration plots using OS nomogram model. (A) Evaluation of calibration plot based on OS of training dataset in 1-year; (B) evaluation of calibration plot based on OS of training dataset in 3-year; (C) evaluation of calibration plot based on OS of training dataset in 5-year; (D) evaluation of calibration plot based on OS of validation dataset in 1-year; (E) evaluation of calibration plot based on OS of validation dataset in 3-year; (F) evaluation of calibration plot based on OS of validation dataset in 5-year. OS, overall survival.

differences in between. First, the period of included cases in Zhou et al. was between 2005 and 2014 while the prognostic data of resources from our study were updated to 2016. Second, the inclusion criteria were different with only 1,445 cases in Zhou et al. results while we included 4,414 cases. The definition of ELGC was above 75 in Zhou et al. while above 70 years old in our study. In fact, we have solid evidence to support the definition (10-12). Third, both OS and CSS study were included in our study compared to Zhou et al., enhancing the distinct clinical values of the nomogram models in our results. Moreover, compared to the variables of nomogram in Zhou et al., sex, age, race, lung metastasis and tumor size also demonstrated remarkable influences on nomogram of OS, especially age itself. We believe the nomogram to predict the prognosis of ELGC opens up an opportunity to notice the significant role of age with much more subtle classification, such as patients between $70-75$ years old, between $76-85$ years old and patients $\geq 86$ years old.

The limitation of this study was, however, lack of another independent clinical datasets to external validation. The predictive power of newly established nomogram required 
A

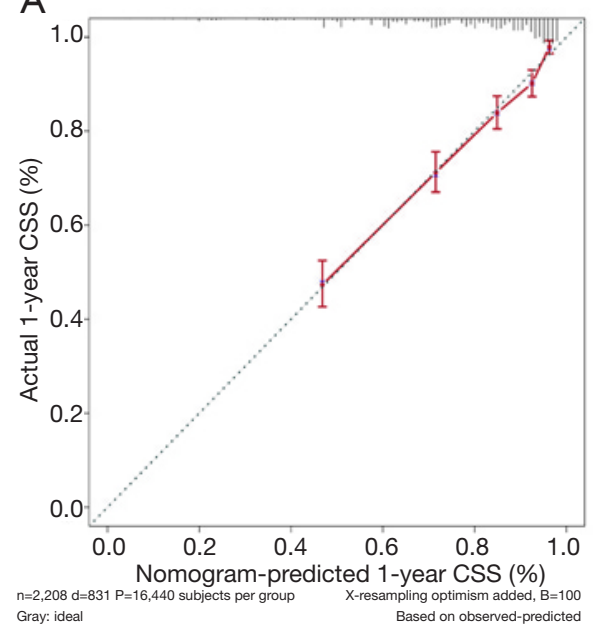

D

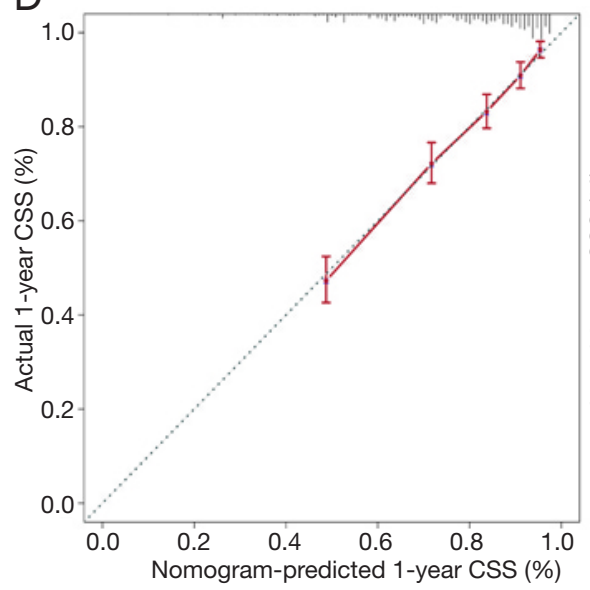

B

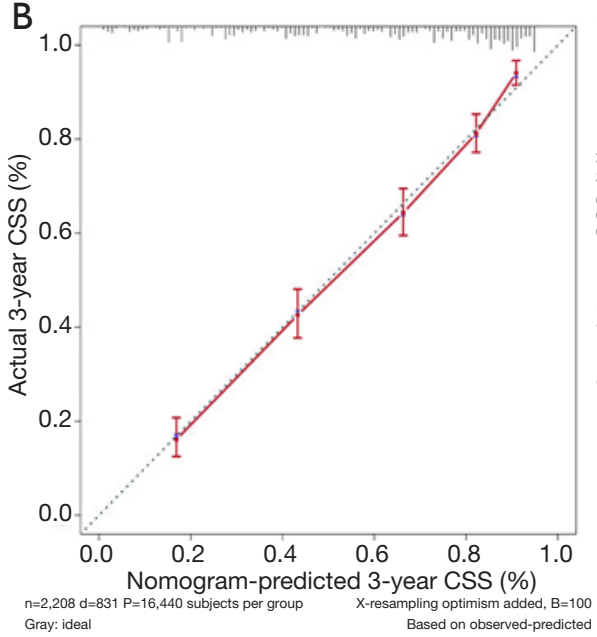

$\mathrm{E}$

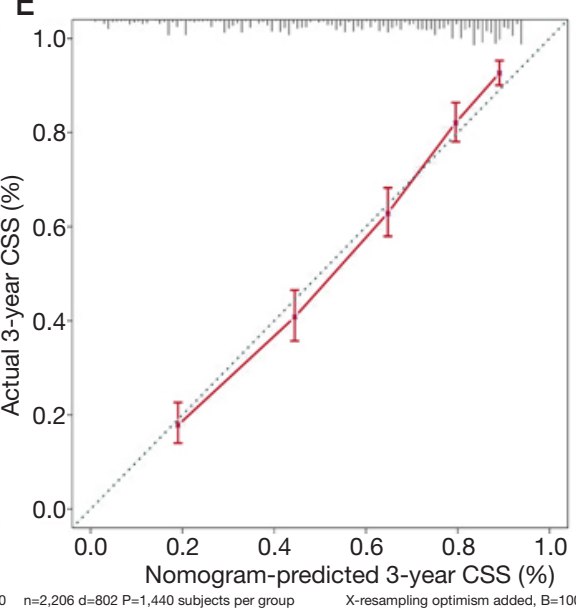

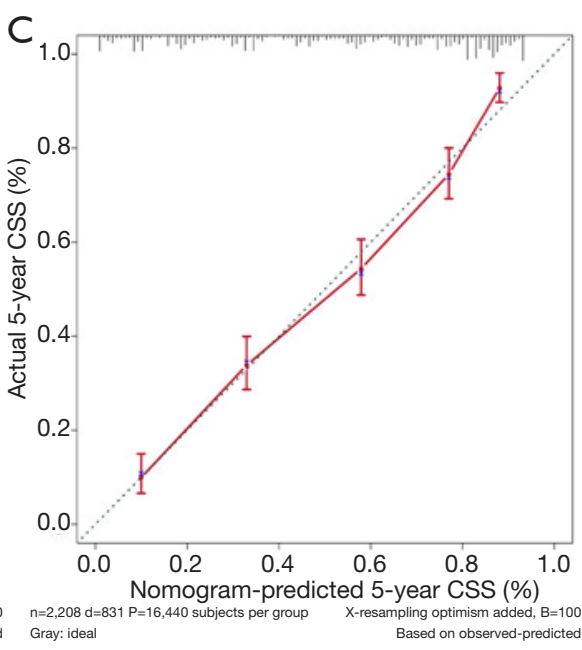

$\mathrm{F}$

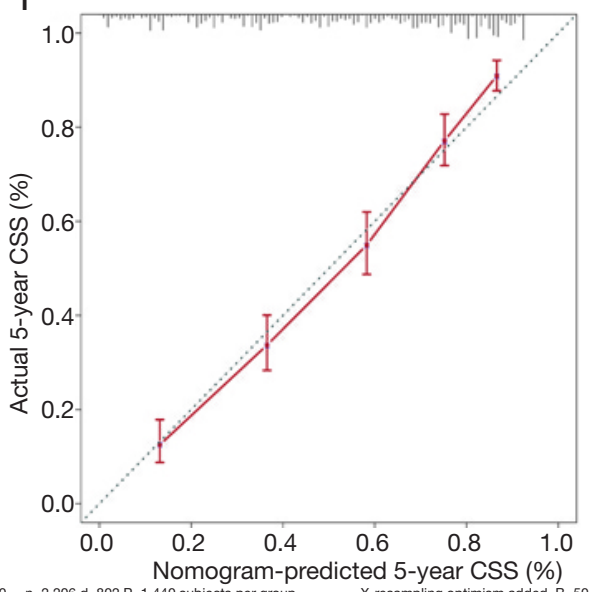

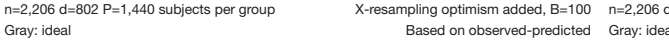

Based on observed-predicted $\quad \mathrm{n}=2,206 \mathrm{~d}=802$

mpling optimism added, $\mathrm{B}=500$

Figure 6 Evaluation of calibration plots using CSS nomogram model. (A) Evaluation of calibration plot based on CSS of training dataset in 1-year; (B) evaluation of calibration plot based on CSS of training dataset in 3-year; (C) evaluation of calibration plot based on CSS of training dataset in 5-year; (D) evaluation of calibration plot based on CSS of validation dataset in 1-year; (E) evaluation of calibration plot based on CSS of validation dataset in 3-year; (F) evaluation of calibration plot based on CSS of validation dataset in 5-year. CSS, cancerspecific survival.

Table 5 C-indexes for the nomograms and AJCC stage in patients with ELGC

\begin{tabular}{|c|c|c|c|c|c|c|}
\hline Survival & \multicolumn{3}{|c|}{ Training set } & \multicolumn{3}{|c|}{ Validation set } \\
\hline \multicolumn{7}{|l|}{ os } \\
\hline Nomogram & 0.730 & $0.714-0.746$ & Reference & 0.735 & $0.719-0.751$ & Reference \\
\hline AJCC stage & 0.698 & $0.681-0.715$ & $<0.001$ & 0.705 & $0.688-0.722$ & $<0.001$ \\
\hline Nomogram & 0.765 & $0.748-0.782$ & Reference & 0.759 & $0.742-0.776$ & Reference \\
\hline AJCC stage & 0.742 & $0.724-0.760$ & 0.012 & 0.747 & $0.729-0.765$ & 0.060 \\
\hline
\end{tabular}

ELGC, elderly gastric cancer; OS, overall survival; HR, hazard ratio; $\mathrm{Cl}$, confidence interval. 

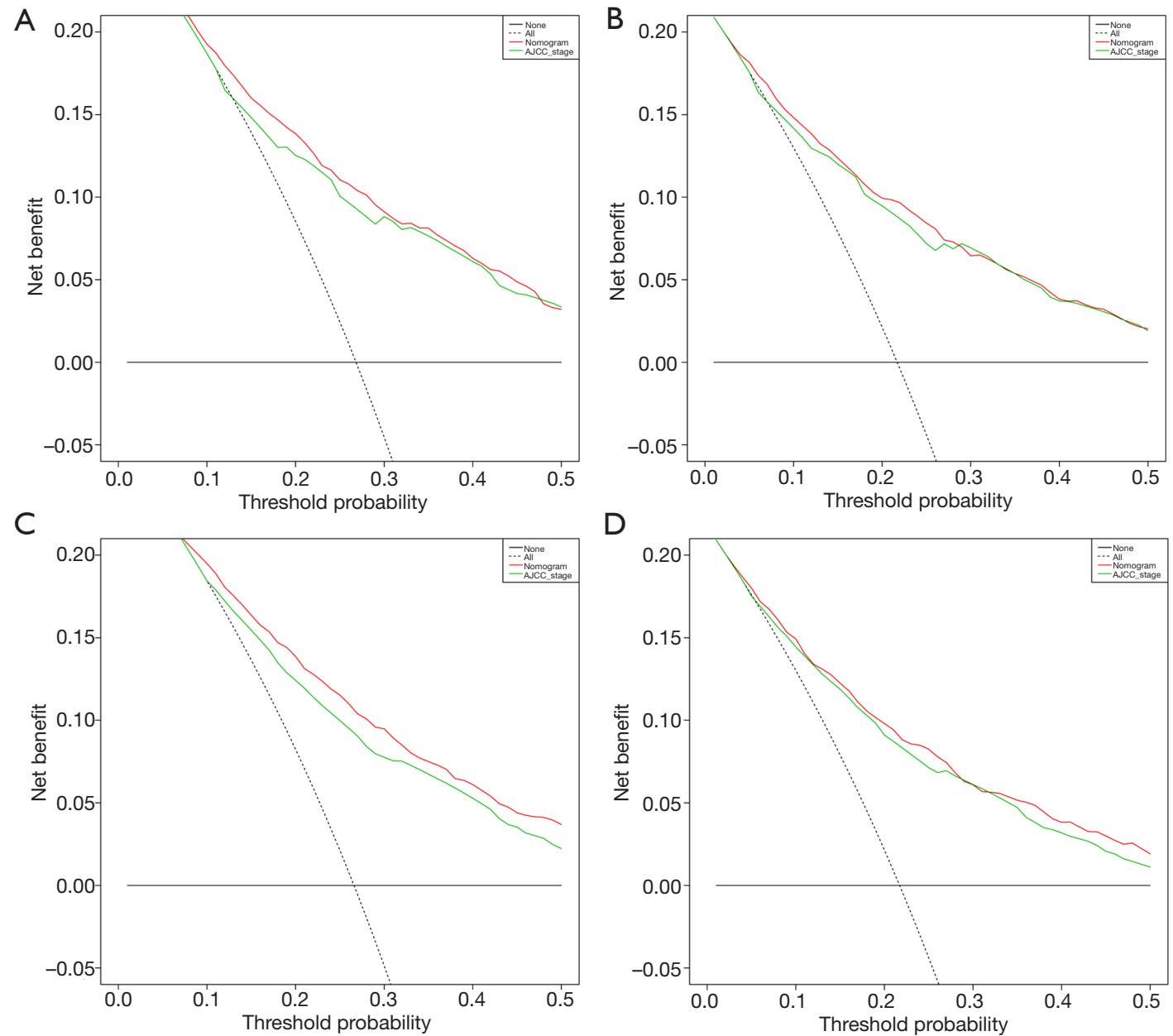

Figure 7 Display of decision curve analysis (DCA) of nomograms models both for OS and CSS. (A) DCA in nomogram using OS training dataset; (B) DCA in nomogram using OS validation set; (C) DCA in nomogram using CSS training set; (D) DCA in nomogram using CSS validation set. Green: AJCC stage; red: nomogram. OS, overall survival; CSS, cancer-specific survival. 
A

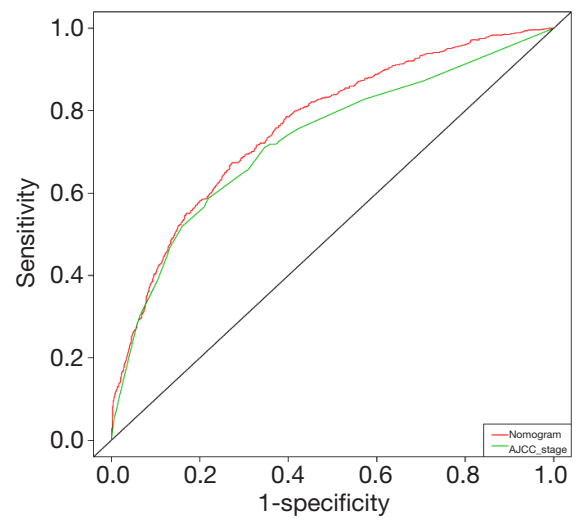

D

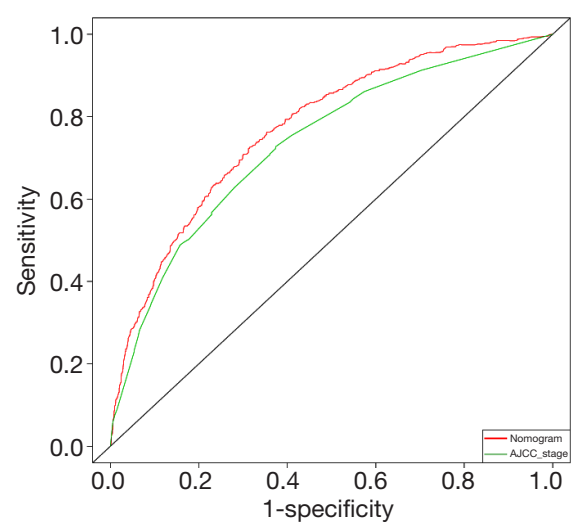

B

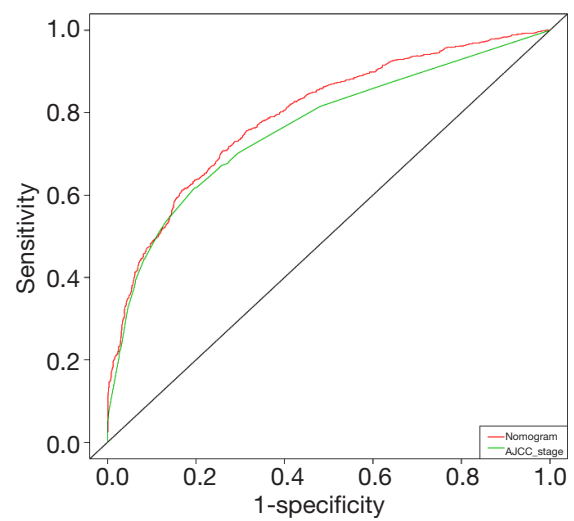

$\mathrm{E}$

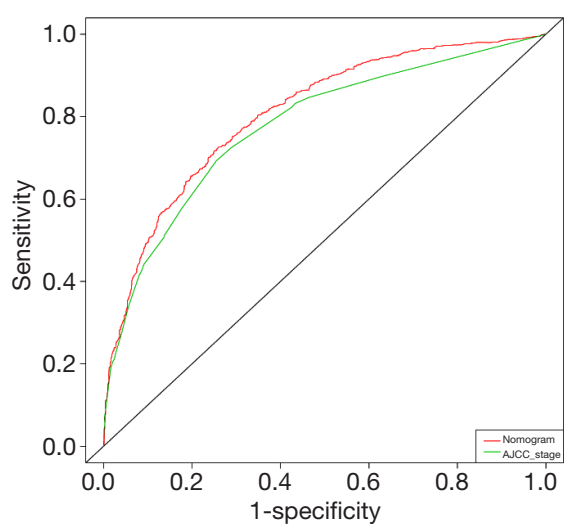

C

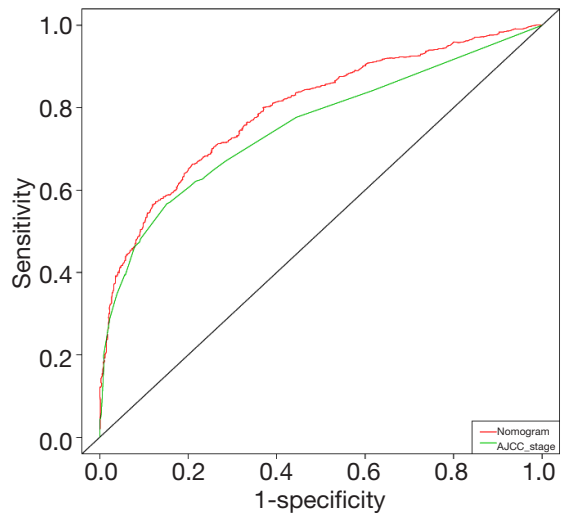

$\mathrm{F}$

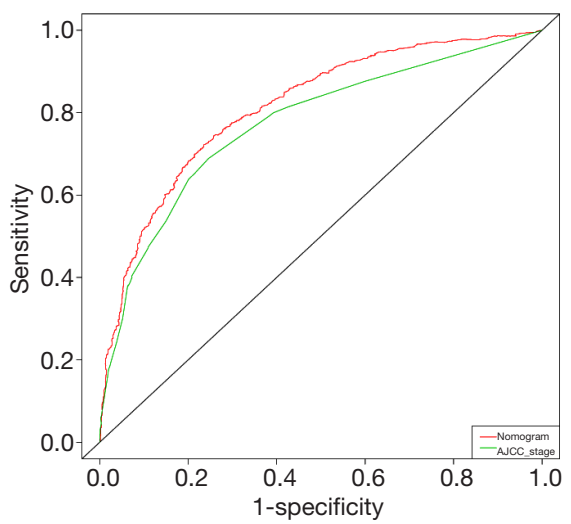

Figure 8 Receiver operating characteristics curve (ROC) comparison of OS nomogram and AJCC TNM stage. (A) ROC of nomogram using OS of train dataset in 1-year; (B) ROC of nomogram using OS of train dataset in 3-year; (C) ROC of nomogram using OS of train dataset in 5-year; (D) ROC of nomogram using OS of validation dataset in 1-year; (E) ROC of nomogram using OS of validation dataset in 3 -year; (F) ROC of nomogram using OS of validation dataset in 5-year. OS, overall survival. 
A

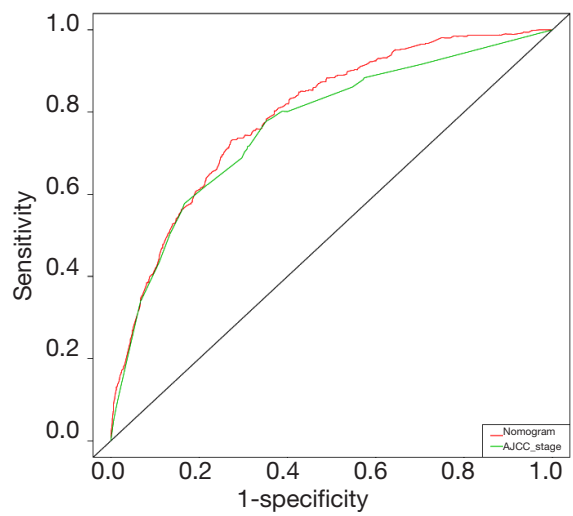

D

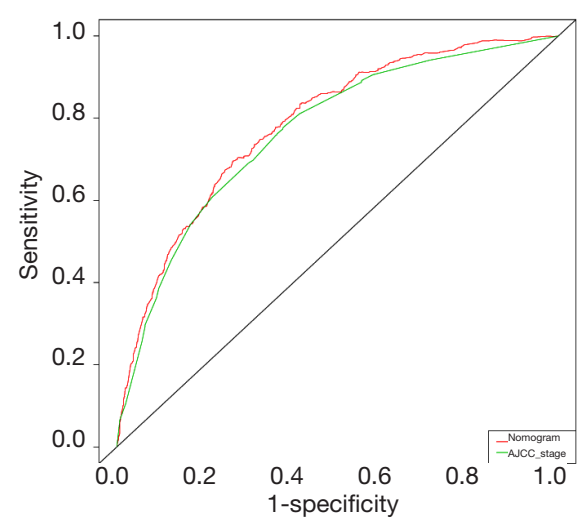

B

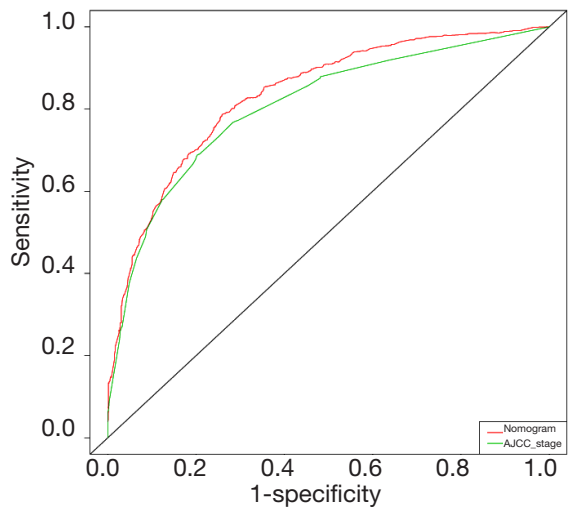

$\mathrm{E}$

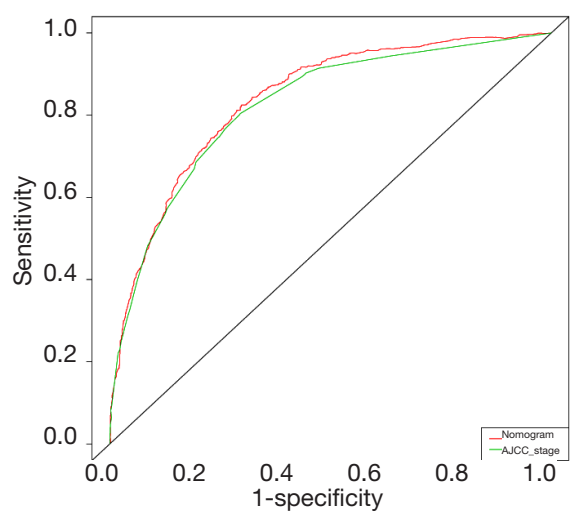

C

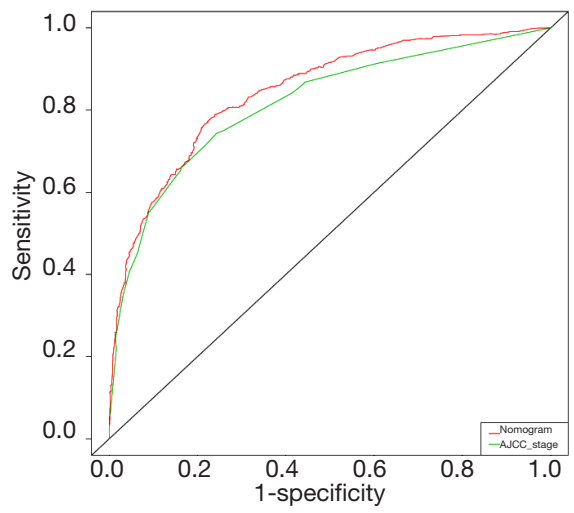

$\mathrm{F}$

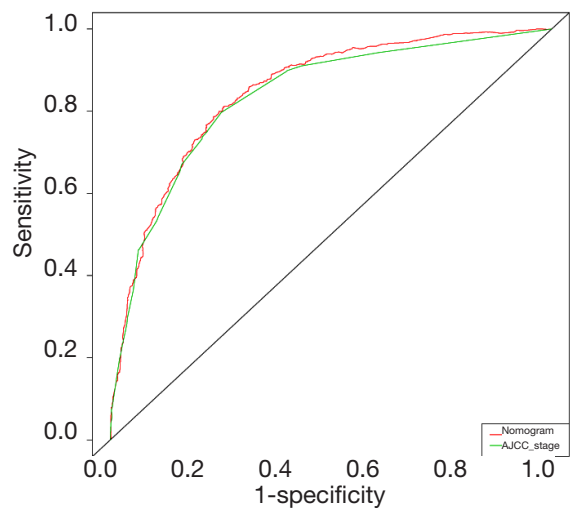

Figure 9 Receiver operating characteristics curve (ROC) comparison of CSS nomogram and AJCC TNM stage. (A) ROC of nomogram using CSS of train dataset in 1-year; (B) ROC of nomogram using CSS of train dataset in 3-year; (C) ROC of nomogram using CSS of train dataset in 5-year; (D) ROC of nomogram using CSS of validation dataset in 1-year; (E) ROC of nomogram using CSS of validation dataset in 3-year; (F) ROC of nomogram using CSS of validation dataset in 5-year. CSS, cancer-specific survival. 
external validation and further refinement.

\section{Conclusions}

This study established and validated novel nomogram models of OS and CSS for ELGC based on population dataset.

\section{Acknowledgments}

Funding: None.

\section{Footnote}

Reporting Checklist: The authors have completed the TRIPOD reporting checklist. Available at http://dx.doi. org/10.21037/jgo-20-536

Conflicts of Interest: Both authors have completed the ICMJE uniform disclosure form (available at http://dx.doi. org/10.21037/jgo-20-536). The authors have no conflicts of interest to declare.

Ethical Statement: The authors are accountable for all aspects of the work in ensuring that questions related to the accuracy or integrity of any part of the work are appropriately investigated and resolved. The study was conducted in accordance with the Declaration of Helsinki (as revised in 2013).

Open Access Statement: This is an Open Access article distributed in accordance with the Creative Commons Attribution-NonCommercial-NoDerivs 4.0 International License (CC BY-NC-ND 4.0), which permits the noncommercial replication and distribution of the article with the strict proviso that no changes or edits are made and the original work is properly cited (including links to both the formal publication through the relevant DOI and the license). See: https://creativecommons.org/licenses/by-nc-nd/4.0/.

\section{References}

1. Prashanth Rawla, Adam Barsouk. Epidemiology of gastric cancer: global trends, risk factors and prevention. Prz Gastroenterol 2019;14:26-38.

2. Thrift AP, El-Serag HB. Burden of Gastric Cancer. Clin Gastroenterol Hepatol 2020;18:534-42.

3. Bray F, Ferlay J, Soerjomataram I, et al. Global cancer statistics 2018: GLOBOCAN estimates of incidence and mortality worldwide for 36 cancers in 185 countries. CA Cancer J Clin 2018;68:394-424.

4. Ahmed N. 23 years of the discovery of Helicobacter pylori: is the debate over? Ann Clin Microbiol Antimicrob 2005;4:17.

5. Peng YC, Huang LR, Lin CL, et al. Association between proton pump inhibitors use and risk of gastric cancer in patients with GERD. Gut 2019;68:374-6.

6. Nagtegaal ID, Odze RD, Klimstra D, et al. The 2019 WHO classification of tumours of the digestive system. Histopathology 2020;76:182-8.

7. Cancer Genome Atlas Research Network. Comprehensive molecular characterization of gastric adenocarcinoma. Nature 2014;513:202-9.

8. Anderson WF, Camargo MC, Fraumeni JF Jr, et al. Agespecific trends in incidence of noncardia gastric cancer in US adults. JAMA 2010;303:1723-8.

9. Yu C, Zhang Y. Development and validation of prognostic nomogram for young patients with gastric cancer. Ann Transl Med 2019;7:641.

10. Saif MW, Makrilia N, Zalonis A, et al. Gastric cancer in the elderly: an overview. Eur J Surg Oncol 2010;36:709-17.

11. Kitamura K, Yamaguchi T, Taniguchi H, et al. Clinicopathological characteristics of gastric cancer in the elderly. Br J Cancer 1996;73:798-802.

12. Liang YX, Deng JY, Guo HH, et al. Characteristics and prognosis of gastric cancer in patients aged $\geq 70$ years. World J Gastroenterol 2013;19:6568-78.

13. Roberto M, Botticelli A, Strigari L, et al. Prognosis of elderly gastric cancer patients after surgery: a nomogram to predict survival. Med Oncol 2018;35:111.

14. Wang SJ, Emery R, Fuller CD, et al. Conditional survival in gastric cancer: a SEER database analysis. Gastric Cancer 2007;10:153-8.

15. Dikken JL, Baser RE, Gonen M, et al. Conditional probability of survival nomogram for 1-, 2-, and 3-year survivors after an R0 resection for gastric cancer. Ann Surg Oncol 2013;20:1623-30.

16. Eil R, Diggs BS, Wang SJ, et al. Nomogram for predicting the benefit of neoadjuvant chemoradiotherapy for patients with esophageal cancer: A SEER-Medicare analysis. Cancer 2014;120:492-8.

17. Hayat MJ, Howlader N, Reichman ME, et al. Cancer statistics, trends, and multiple primary cancer analyses from the Surveillance, Epidemiology, and End Results (SEER) Program. Oncologist 2007;12:20-37.

18. Camp RL, Dolled-Filhart M, Rimm DL. X-tile: a new 
bio-informatics tool for biomarker assessment and outcome-based cut-point optimization." Clin Cancer Res 2004;10:7252-9.

19. Qiu M, Yang D, Xu R. Impact of marital status on survival of gastric adenocarcinoma patients: Results from the Surveillance Epidemiology and End Results (SEER) Database. Sci Rep 2016;6:21098.

20. Ma T, Wu ZJ, Xu H, et al. Nomograms for predicting

Cite this article as: Zhang Y, Yu C. Development and validation of a Surveillance, Epidemiology, and End Results (SEER)-based prognostic nomogram for predicting survival in elderly patients with gastric cancer after surgery. J Gastrointest Oncol 2021;12(2):278-296. doi: 10.21037/jgo-20-536 survival in patients with metastatic gastric adenocarcinoma who undergo palliative gastrectomy. BMC Cancer 2019;19:852.

21. Zhou X, Ding Y, Wang Y, et al. Development and Validation of a Prognostic Nomogram to Predict Overall Survival of Elderly Patients with Gastric Cancer. Research Square 2020. doi: 10.21203/rs.3.rs-36638/v1. 